\title{
Absence of unidentified CAG repeat expansion in patients with Huntington's disease-like phenotype
}

\author{
I Vuillaume, P Meynieu, S Schraen-Maschke, A Destée, B Sablonnière
}

\begin{abstract}
Huntington's disease (HD) is an autosomal dominant neurodegenerative disorder caused by an expanded (CAG)n repeat on the huntingtin gene. It is characterised by motor, psychiatric and cognitive disturbances. Diagnosis can be confirmed by direct genetic testing, which is highly sensitive and specific and is now considered definitive. This study focused on 21 patients presenting with a clinical phenotype showing strong similarity to $\mathrm{HD}$, but who do not have an expanded CAG in the huntingtin gene. However, other possible diagnoses could be evoked for most of them. Seven patients (3.5\% of our cohort) could be considered as phenocopies of HD with no alternative diagnosis. Samples were screened for other triplet repeat diseases with similar presentation (DRPLA, SCA-1, SCA-2, SCA-3, SCA-6, and SCA-7) and were all negative. The repeat expansion detection technique (RED) was used to detect uncloned CAG repeat expansions and samples were also analysed by polymerase chain reaction for expansions of the polymorphic CAGERDA-1 and CTG18.1 trinucleotide repeats. RED expansion ( $>40$ repeats) was detected in only one patient. The results suggest that unstable CAG/CTG repeat expansions corresponding to known or unknown sequences are not involved in the aetiology of HD-like disorders. It is hypothesised that some of these phenocopies could correspond to mutations in other unidentified genes with other unstable repeats (different from CAG) or in unknown genes with other mutations. (F Neurol Neurosurg Psychiatry 2000;68:672-675)
\end{abstract}

Keywords: Huntington's disease-like; CAG repeat expansion

Huntington's disease (HD) is an autosomal dominant neurodegenerative disorder caused by an expanded (CAG)n repeat in the huntingtin gene. The HD phenotype includes involuntary, choreiform movements, and cognitive and psychiatric disturbances, arising in patients, typically in mid-adult life. ${ }^{12}$ In the past, diagnosis used to be on the basis of family history, characteristic clinical features, and on the general course of the disease. Since the identification in 1993 of the gene responsible for HD, diagnosis can be now confirmed by direct genetic testing, which is both highly sensitive and specific. ${ }^{3}$ DNA testing has made it possible to find out whether patients definitely have or do not have HD, even in the absence of family history. Moreover, the absence of the characteristic CAG expansion can definitively exclude diagnosis of HD in some patients with both HD-like symptoms and possible family history. Differential diagnosis includes disorders such as benign familial chorea, Wilson's disease, Sydenham's chorea, and neuroacanthocytosis that may be distinguished from HD according to clinical features and to further laboratory investigations. Interestingly, a growing number of neurodegenerative diseases, which could mimic some clinical features of HD, have been associated with other triplet repeat expansions: dentatorubro-pallidoluysian atrophy and spinocerebellar ataxias SCA-1, SCA-2, SCA-3, SCA-6, and SCA-7.(See Robitaille et $a l^{4}$ and references therein), for which genes have been identified and for which DNA tests are available. In the recent past, a method named repeat expansion detection (RED) has been used to search for large potentially unstable trinucleotide repeats. ${ }^{5}$ In RED, a thermostable ligase is used in a cyclic procedure to detect repeat expansions directly from genomic DNA without information on their location in the genome. This assay has previously shown evidence of an increased number of people with CAG repeat expansions among affected members of pedigrees ascertained for bipolar disorders, ${ }^{6}$ schizophrenia, ${ }^{7}$ and hereditary spastic paraplegia. ${ }^{8}$ However, identification of large repeats through the RED technique does not necessarily imply their responsibility in illness. Searching for new triplet repeat candidate genes, two expanded repeat loci, ERDA-1 on chromosome 17 and CTG18.1 on chromosome 18, were recently identified $^{910}$ in normal people. A few patients with HD-like features but without the expected mutation, as shown in previous studies, may represent phenocopies; the genetic cause for their phenotype lying in another CAG expansion in an as yet unknown gene. In this regard, we reviewed 21 isolated patients with probable or possible HD on clinical grounds and imaging findings, but who do not have an expanded CAG in the huntingtin gene. We 
Table 1 Summary of clinical and MRI findings of patients from the first group

\begin{tabular}{|c|c|c|c|c|c|}
\hline $\begin{array}{l}\text { Patient } \\
\text { No }\end{array}$ & $\begin{array}{l}\text { Age at } \\
\text { onset }\end{array}$ & Main clinical features & Other clinical features & $M R I$ & Possible diagnosis \\
\hline $\mathrm{H} 5$ & 64 & Chorea, dementia, depression & & Corticosubcortical atrophy & HD-like \\
\hline $\mathrm{H} 48$ & 47 & Chorea, dementia, behav disturb & Obsessive features & Caudate atrophy & HD-like \\
\hline H100 & 62 & Chorea, dementia & Frontal release signs & Corticosubcortical atrophy & HD-like \\
\hline $\mathrm{H} 102$ & 66 & Chorea, dementia & Frontal release signs & $\begin{array}{l}\text { Lentiform nucleus atrophy } \\
\text { Corticosubcortical atrophy, }\end{array}$ & HD-like \\
\hline $\mathrm{H} 122$ & 56 & Chorea, dementia, depression & & pallidal hyperintensities & HD-like \\
\hline $\mathrm{H} 154$ & 40 & $\begin{array}{l}\text { Chorea, dementia, depression } \\
\text { Behav disturb }\end{array}$ & Frontal release signs & ND & HD-like \\
\hline H26 & 60 & Chorea, dementia, behav disturb & Ataxia & Cerebellar and caudal atrophy & HD-like/cerebellar disorder \\
\hline H139 & 30 & $\begin{array}{l}\text { Choreaoathetosis, dysarthria, } \\
\text { dementia, behav disturb. }\end{array}$ & $\begin{array}{l}\text { Pyramidal signs, hypertension, } \\
\text { Angor, ischaemic cardiopathy }\end{array}$ & ND & HD-like \\
\hline H150 & 64 & Chorea, dementia, behav disturb & & Cortical and cerebellar atrophy & HD-like/cerebellar disorder \\
\hline H155 & 46 & Chorea, dementia, behav disturb & Ataxia & Cortical and cerebellar atrophy & HD-like/cerebellar disorder \\
\hline H165 & 49 & Chorea, dementia & Ataxia & & HD-like/cerebellar disorder \\
\hline
\end{tabular}

behav disturb=Behavioural disturbances; $\mathrm{ND}=$ not determined.

intended to determine whether or not patients with an HD-like disorder could be traced to other known or unknown triplet repeat diseases.

\section{Patients and methods}

PATIENTS

The medical records of all patients diagnosed as having HD or possible HD over the period 1995 to 1998 at the Clinique Neurologique in Lille were reviewed. Clinical details were based on extensive records documenting neurological examination and special investigations such as MRI, copper studies, peripheral smears, and muscle biopsies. All patients gave their informed consent for DNA analysis.

GENETIC ANALYSIS

Analyses of DNA were based on genomic DNA isolated from leucocytes and run in duplicate and separately to prevent laboratory errors. The CAG repeat length was determined using the polymerase chain reaction (PCR) with primers HD1 and HD2 as well as with primers HD1 and HD3 just flanking the CAG repeat, as previously described. ${ }^{11} \mathrm{HD}$ negative samples from symptomatic patients were screened for other triplet repeat diseases with similar presentation; DRPLA repeats were amplified using primers B37-1 and B37-2; SCA-1 using primers Rep-1 and Rep-2; SCA-2 using primers UH10 and UH13; SCA-3 using primers MJD52 and MJD25; and SCA-6 using primers $\mathrm{S} 5 \mathrm{~F} 1$ and S5R1. Allele sizes were measured as described. ${ }^{11}$ We used the RED technique to detect uncloned trinucleotide expansions as described by Schalling et al. ${ }^{5}$ The same samples were then analysed for expansions of the polymorphic CAG ERDA-1 trinucleotide repeat by use of PCR, using conditions precisely described elsewhere. ${ }^{9}$ All samples which were RED positive (expansions $>40$ repeats) and CAG ERDA-1 negative were thereafter analysed for expansion at the CTG18.1 locus, using a previously described PCR protocol. ${ }^{10}$

\section{Results}

CASE REPORTS

Out of 200 patients assessed in our Lille cohort for diagnostic confirmation, 32 patients did not have expansion of the CAG repeat in the huntingtin gene. We excluded from this study 11 patients clearly presenting a likely cause of sporadic chorea such as drug induced chorea or stroke related chorea with sudden onset. We further analysed 21 patients from different families who could be considered as having a possible hereditary neurodegenerative disease similar to HD. All these patients had a possible family history which was ascertained for 10 of them. The acanthocytosis screen, copper studies, and blood and CSF biochemistry screening were not informative. Diagnosis was reconsidered according to their history, clinical presentation, and neuroradiological findings. Eleven patients (first group) presented clinical features very suggestive of $\mathrm{HD}$ as summarised in table 1. Atrophy of the caudate or lentiform nuclei on MRI further supported an HD-like diagnosis in two patients. Interestingly, onset occurred significantly later in life (53 years) than in typical HD (42 years in total cohort). For seven of them, clinical features are consistent with HD and appear as possible phenocopies with no alternative diagnosis. For four of them, the presence of ataxia and cerebellar atrophy may be suggestive of a cerebellar disease. In the second group, 10 patients did not seem to have an HD-like disease. Two of them proved to have benign hereditary chorea and progressive myoclonus epilepsia was proposed for two other patients presenting with epilepsy, myoclonia, and dementia. Vascular chorea was considered for three patients based on a non-progressive chorea; vascular lesions were disclosed on imaging and patient history of vascular disease. The last three patients had a disorder which could not be clearly classified.

\section{GENETIC ANALYSIS}

Table 2 shows that all patients studied were negative for the HD gene expansion giving a CAG repeat ranging from 10 to 28 . As other

Table 2 Genetic analysis

\begin{tabular}{lllll}
\hline $\begin{array}{l}\text { Patient } \\
\text { No }\end{array}$ & $\begin{array}{l}(C A G) n \\
\text { HD gene }\end{array}$ & RED* & ERDA-1* & CTG18.1* \\
\hline H5 & $21 / 16$ & 40 & $16 / 10$ & ND \\
H48 & $21 / 17$ & 40 & $31 / 20$ & ND \\
H100 & $19 / 10$ & 60 & $30 / 21$ & $15 / 8$ \\
H102 & $27 / 19$ & 40 & $31 / 21$ & ND \\
H122 & $21 / 16$ & 40 & $21 / 7$ & ND \\
H154 & $28 / 20$ & 40 & $16 / 10$ & ND \\
H26 & $19 / 10$ & 40 & $21 / 21$ & ND \\
H139 & $26 / 20$ & 40 & $23 / 20$ & ND \\
H150 & $17 / 15$ & 40 & $20 / 15$ & ND \\
H155 & $20 / 19$ & 40 & $10 / 10$ & ND \\
H165 & $19 / 18$ & 40 & $21 / 21$ & ND \\
\hline
\end{tabular}

${ }^{\star}$ Number of CAG/CTG repeats; ND=not determined 


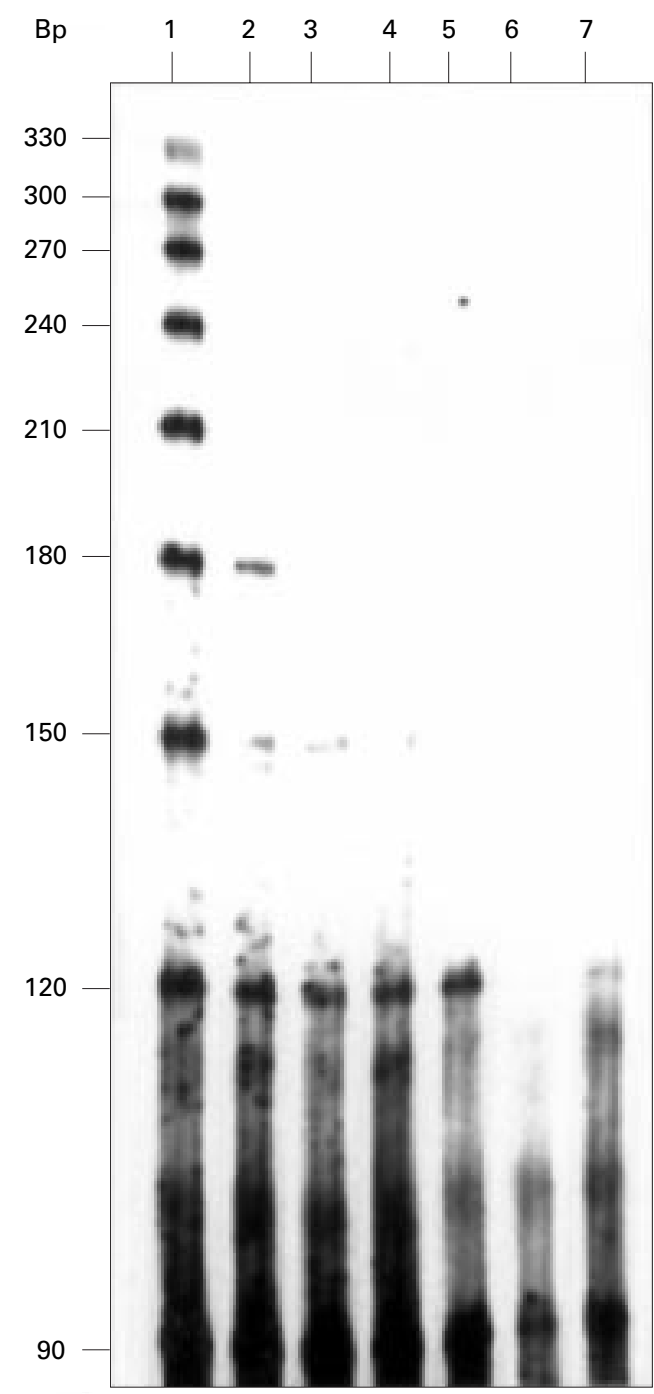

$C A G$ repeat sizes of ligation products detected by RED analysis. Lane 1 shows a ligation product of 110 repeats obtained from a patient with myotonic dystrophy as a positive control. The other lanes show expansions of different sizes from 30 to 60 repeats.

triplet repeat disorders are characterised by HD-like symptoms, patients were therefore tested for different known mutations and were found negative for every characterised cerebellar ataxia gene (SCA-1, SCA-2, SCA-3, and SCA-6) as well as for the DRPLA gene. Thereafter, to search for other unstable CAG expansions, we used the RED technique (figure). Such expansions, detected above a threshold value of 40 repeats, were found in only one out of 11 patients of the first group. In this case (H100), an expansion was found in a patient presenting with a possible phenocopy of HD ; but the RED results could not be explained by an increase in trinucleotide number, neither at the ERDA-1 nor at the CTG18.1 locus.

\section{Discussion}

Between 1995 and 1998, of 200 patients in our Lille cohort who had been suspected of having HD, 32 (16\% of our cohort) had CAG repeat lengths in the normal range (10-28 repeats). In previous studies, ${ }^{12}{ }^{13}$ less than $3 \%$ of patients tested for diagnosis confirmation were actually gene negative. This discrepancy could be accounted for by a significant shift in the prescription of the test. Whereas most of the tests used to be carried out for diagnostic confirmation in families with long established HD, an increasing number of them now apply to patients presenting with substantial diagnostic problems, conveyed either by atypical clinical or neuroradiological signs or by an unclear family history. The easily available DNA test encouraged physicians to confirm or rule out this diagnosis in such patients. In our study, seven patients could be phenocopies of HD, although they did not meet every criteria defining a HD phenocopy (typical findings on postmortem examination and a family history consistent with autosomal dominant transmission). A few patients have been considered as phenocopies, with neuropathological changes for four of them. ${ }^{12}{ }^{1415}$ Negative gene tests for DRPLA and spinocerebellar ataxias were reported by Rosenblatt et $a l^{13}$ in four patients described as potential phenocopies. However, screening for triplet repeat expansions was not reported. In this limited study we tried to consider whether patients with HD-like disorders could be traced to other triplet repeat diseases. Accordingly, we endeavoured to complete our study to this purpose. The RED technique provides here an interesting strategy for searching for unknown unstable CAG/CTG repeat expansions. However, the performance of this method does not allow detection of small expansions (such as those found in SCA6) as the threshold is about 40 repeats. ERDA-1 and CTG18.1 loci represent polymorphic and unstable sequences which may explain RED scores of 40 repeats or more. Therefore, detection of positive RED scores in patients who lack expansions at both the ERDA-1 and CTG18.1 loci has much higher likelihood of being disease related. Ten out of 11 patients did not exhibit any unstable CAG/ CTG repeat expansion as their RED product sizes were lower than 40 repeats. Only one patient with a RED product size of 60 repeats was found that could not be explained by expansion at ERDA-1 or CTG18.1 loci.

An increasing number of hereditary neurodegenerative disorders have been attributed to triplet repeat expansions in various genes. Our results suggest that unstable CAG or CTG expansions corresponding to uncloned or cloned sequences are probably not associated with the aetiology of rare HD-like diseases with possible family history. This study was, however, performed in a limited series of such cases and further work will be needed to confirm these findings in a larger cohort. We think that some HD-like disorders may be caused by mutations other than unstable CAG triplet repeats, such as was recently described on chromosome 20. in a Swedish family. ${ }^{16}$ However, we cannot exclude the possibility that other unstable repeats (different from CAG), in other unidentified genes may be implicated. 
We thank Guy Bocquillon, Patrick Devos, Christiane Marzys, and Carole Verlez for their skilful technical assistance. This work was supported by grants from CHRU of Lille.

1 Hayden MR. Huntington's chorea. New York: Springer Verlag, 1981 .

2 Harper PS. Huntington's disease. London: Saunders, 1991

3 Kremer B, Goldberg P, Andrew SE, et al. A worldwide study

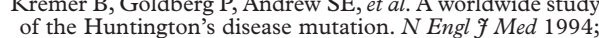
of the Hun

4 Robitaille Y, Lopes-Cendes I, Becher M, et al. The neuropathology of CAG repeat diseases: review and update of genetic and molecular features. Brain Pathol 1997,7:901-26.

5 Schalling M, Hudson TJ, Buetow KH, et al. Direct detection of novel expanded trinucleotide repeat in the human genome. Nat Genet 1998;62:1548-51.

6 Lindblad K, Nylander PO, De Bruyn A, et al. Detection of expanded CAG repeats in bipolar affective disorder using the repeat expansion detection (RED) method. Neurobiol Dis 1996;2:55-62.

7 Morris AG, Gaitonde E, McKenna PJ, et al. CAG repeat expansions and schizophrenia: association with disease in females and with early age-at-onset. Hum Mol Genet 1995; 4:1957-61.

8 Nielsen JE, Koefed P, Abell K, et al. CAG repeat expansion in autosomal dominant pure spastic paraplegia linked to chromosome 2p21-p24. Hum Mol Genet 1997;6:1811-6.
9 Ikeuchi T, Sanpei K, Takano $\mathrm{H}$, et al. A novel long and unstable CAG/CTG trinucleotide repeat on chromosome 17q. Genomics 1998;49:321-6.

10 Breschel TS, McInnis MG, Margolis RL, et al. A novel heritable expanding CTG repeat in an intron of the SEF2-1 gene on chromosome 18q21.1. Hum Mol Genet 1997;6: 1855-63.

11 Vuillaume I, Schraen S, Rousseaux J, et al. Simple nonisotopic assays for detection of (CAG)n repeat expansions associated with seven neurodegenerative disorders. Diag Mol Pathol 1998;7:174-9.

12 Andrew SE, Goldberg YP, Kremer B, et al. Huntington disease without CAG expansion: phenocopies or error in assignment ? Am f Hum Genet 1994;54:852-63.

13 Rosenblatt A, Ranen NG, Rubinsztein DC, et al. Patients with features similar to Huntington's disease, without CAG expansion in huntingtin. Neurology 1998;51:215-20.

14 Persichetti F, Srinidhi J, Kanaley L, et al. Huntington's disease CAG trinucleotide repeats in pathologically confirmed post-mortem brains. Neurobiol Dis 1994;1:159-66.

15 Xuereb JH, McMillan JC, Snell R, et al. Neuropathological diagnosis and CAG repeat expansion in Huntington's disease. F Neurol Neurosurg Psychiatry 1996;60:78-81.

16 Xiang F, Almqvist EW, Huq M, et al. A Huntington diseaselike neurodegenerative disorder maps to chromosome $20 \mathrm{p}$. Am f Hum Genet 1998;63:1431-8.

\section{NEUROLOGICAL STAMP}

\section{Georg von Békésy (1899-1972)}

Born in 1899 in Budapest of a distinguished family, Georg von Békésy studied at the University of Berne; at the University of Budapest, where he obtained his $\mathrm{PhD}$; and at the University of Münster, where he received an MD with honours. In 1928, Békésy published his first and probably most significant paper on the pattern of vibration in the membranes of the cochlea of the ear. This one paper provided the foundations for his whole career: "I found the inner ear so beautiful under a stereoscopic microscope that I decided I would just stay with that problem. It was the beauty and the pleasure of beauty that made me stick to the ear."

Since that date, he regularily published papers about the theory of hearing, notably how the cochlea functions mechanically. In 1947 he went to the USA and from 1949 was a senior research fellow in psychophysics at Harvard University. In 1961 Georg von Békésy was awarded the Noble Prize in Physiology of Medicine for his discoveries on the physical mechanism of stimulation within the cochlea. Békésy's writings on the main results of his many studies on hearing over the 30 year period $1928-58$ were published in 1960 in a single volume Experiments in Hearing. Békésy died in 1972 in Honolulu. He will be best remembered for inventing the patient controlled recording audiometer, known to all of us as the Békésy audiometer.

Békésy was primarily a physicist. As an investigator he was imaginative, precise, immensely patient, and single minded. He set a new standard for bioacoustical investigation. He became a legend in his own lifetime, and he will always be remembered for his many contributions to otol-

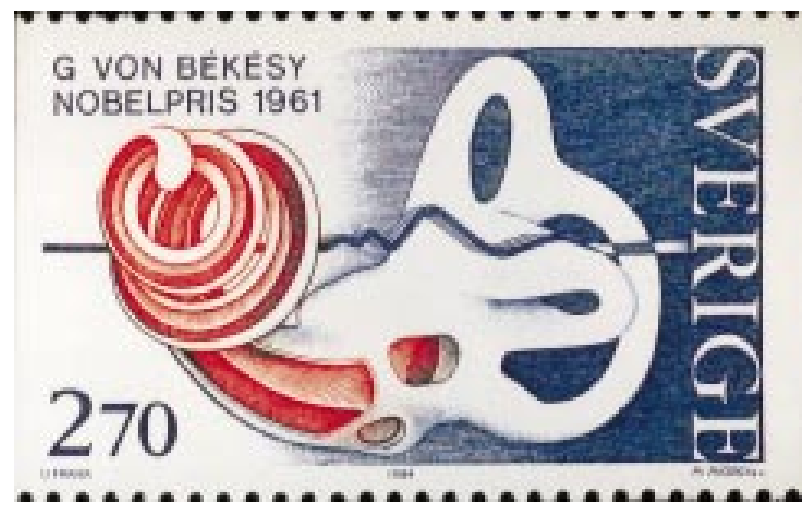

ogy, to auditory physiology, and to psychophysics. He was a solitary worker. One of his rare collaborations was with Julius Lempert, one of the most prominent ear surgeons in the middle of the 20th century. His life was devoted almost as much to art as it was to science; and although his love of art was carefully cultivated, the course of his scientific career was determined almost as much by chance as it was by design.

Békésy was honored philatelically by a stamp issued in Sweden in 1984, to remember his Nobel Prize Award and by a stamp issued in Hungary, his country of origin, in 1988.

ALBERT MUDRY Private practice and ENT Department, University Hospital (CHUV) Lausanne, Avenue de la Gare 6, CH-1003 Lausanne, Switzerland email amudry@worldcom.ch 